\title{
Measuring the contact force of a pantograph by image processing technology
}

\author{
T. Koyama ${ }^{1}$, M. Ikeda ${ }^{1}$, K. Nakamura ${ }^{2}$, S. Tabayashi ${ }^{3}$ \\ \& M. Niwakawa ${ }^{3}$ \\ ${ }^{1}$ Railway Technical Research Institute, Japan \\ ${ }^{2}$ Railway Technical Research Institute, \\ (currently at Central Japan Railway Company), Japan \\ ${ }^{3}$ Meidensha Corporation, Japan
}

\begin{abstract}
A contact force between pantograph and contact line is one of the important criteria to evaluate contact performance. Hence, contact force measuring methods were developed. The currently-used measurement methods use built-in sensors on the panhead. However, these sensors affected the dynamic characteristics of the pantograph. In addition, the methods need data transmitting equipment, because built-in sensors are set under a high-voltage condition. To eliminate these drawbacks, the authors have developed a new contact force measuring method which can replace the all built-in sensors with image processing sensors mounted on the rooftop of the train. To verify measurement accuracy of this method, we performed an excitation test of the pantograph. Experimental results show that the measurement method meets the requirements of the EN standard.

Keywords: contact force, pantograph, catenary, image processing, line sensor camera, EN standard.
\end{abstract}

\section{Introduction}

The contact force between a pantograph and contact line is one of the important criteria to evaluate contact performance $[1,2]$. These days, many studies have been conducted to diagnose overhead catenary system conditions by utilizing contact force measurements [3]. Hence, contact force measuring methods were developed [4-7]. 
In the currently-used methods, the contact force is measured by using built-in sensors such as loadcells and accelerometers set in a panhead. These methods, however, have some serious problems. That is, these sensors affect the dynamic characteristics of the pantograph. In addition, the method requires data transmitting equipment, because these sensors are set under a high-voltage condition.

To eliminate these drawbacks, authors have developed a new contact force measuring method, which can replace the whole of the built-in sensors with image processing sensors.

\section{The principles of the contact force measurement method}

\subsection{An outline of the contact force measurement method}

Some contact force measurement methods have already been developed. Since many of them are based on the force equilibrium equation, its outline is described in this section [6].

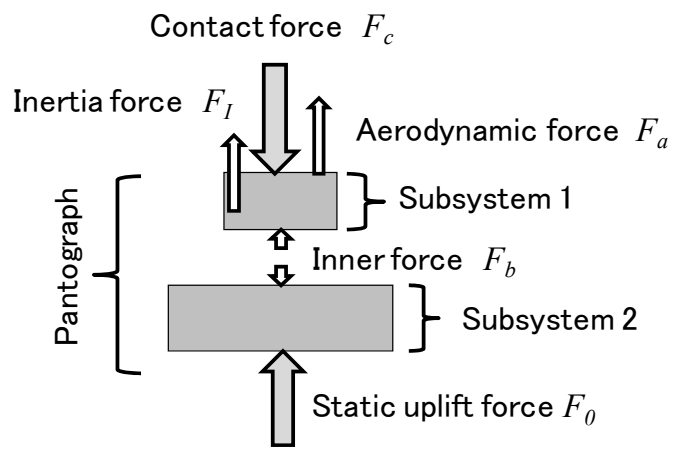

Figure 1: $\quad$ Force equilibrium in a pantograph.

Figure 1 shows a mechanical model of the pantograph. The pantograph is divided into two subsystems: the subsystem 1 corresponds to a panhead, and the subsystem 2 corresponds to an articulated frame and panhead support. Contact force $F_{c}$, inner force $F_{b}$ acting upon the interface between subsystem 1 and subsystem 2, aerodynamic force $F_{a}$ and inertia force $F_{I}$ affecting on Subsystem 1 equilibrate. Thus, we can obtain the force equilibrium equation of subsystem 1.

Eqn. (1) indicates that contact force $F_{c}$ can be evaluated as a summation of $F_{b}$, $F_{a}$, and $F_{I}$.

There is, however, no practical method for measuring aerodynamic force $F_{a}$, while the pantograph is sliding with contact wire. Since the aerodynamic force $F_{a}$ is proportional to the square of relative flow velocity $V_{t}$ against the pantograph, $F_{a}$ can be estimated by eqn. (2).

$$
F_{a}=C_{a} V_{t}^{2}
$$


where, $C_{a}$ is the proportionality constant, which can be obtained by a wind tunnel test.

Hence, only inner force $F_{b}$ and inertia force $F_{I}$ affecting subsystem 1 should be measured in order to evaluate the contact force.

\subsection{Measurement method of the inner force}

The inner force $F_{b}$ consist of spring reaction forces $F_{b s}$ of the panhead supporting springs if the friction force between the panhead and the panhead support can be negligible (See Figure 2). Therefore, the inner force $F_{b}$ can be expressed as follows:

$$
F_{b}=\sum_{i=1}^{2} F_{b s, i} \quad(i=1,2)
$$

In the case of the currently-used contact force measurement method, the inner forces $F_{b s, i}$ are generally measured by built-in sensors such as loadcells or strain gauges set in the panhead $[5,6]$.

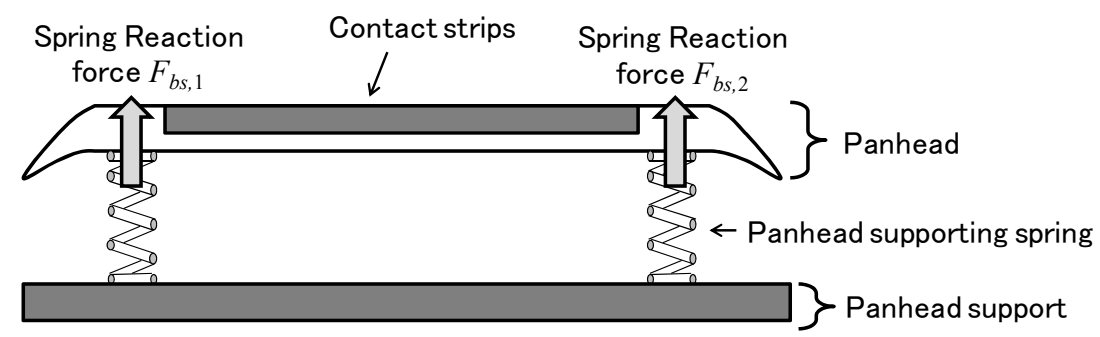

Figure 2: $\quad$ The details of inner force.

\subsection{Measurement method of the inertia force}

Only in low frequency range, the inertia force $F_{I}$ can be evaluated by mass of the panhead and the acceleration $a$ of the panhead.

$$
F_{I}=m a
$$

If the contact force is required to be measured up to high frequency range, the inertia force has to be evaluated by the product of the accelerations of plural positions on the panhead and the corresponding equivalent mass $m_{i}$ [6].

$$
F_{I}=\sum m_{i} a_{i}
$$

In the case of the currently-used contact force measurement method, the accelerations are generally measured by built-in accelerometers set into the panhead $[5,6]$. 


\section{Measuring the contact force by image processing technology}

The authors have developed a new contact force measurement method using the image processing technology with line sensor cameras, by which any built-in sensor is not needed. This chapter explains principle of this method.

\subsection{The principle of the new method}

As noted in chapter 2, if the friction force between the panhead and the panhead support can be negligible, only the spring reaction forces $F_{b s}$ and the inertia force $F_{I}$ have to be measured to obtain the contact force $F_{c}$. According to Hooke's law, the reaction forces $F_{b s}$ are obtained by the product of the spring coefficient and the spring deformation which corresponds to the relative displacement between the panhead and the panhead support. The inertia force $F_{I}$ can be obtained by the product of the mass of the panhead and the acceleration which can be obtained as the second derivative of the displacement of the panhead with respect to time. This means that we can measure the contact force by evaluating displacement of the panhead and panhead support.

\subsection{The displacements measurement method by image processing technology}

A schematic drawing of this method is as shown in Figure 3. Some markers with a black stripe are put on the surface of the panhead and the panhead support to evaluate displacements of the panhead and the panhead support. Images of these

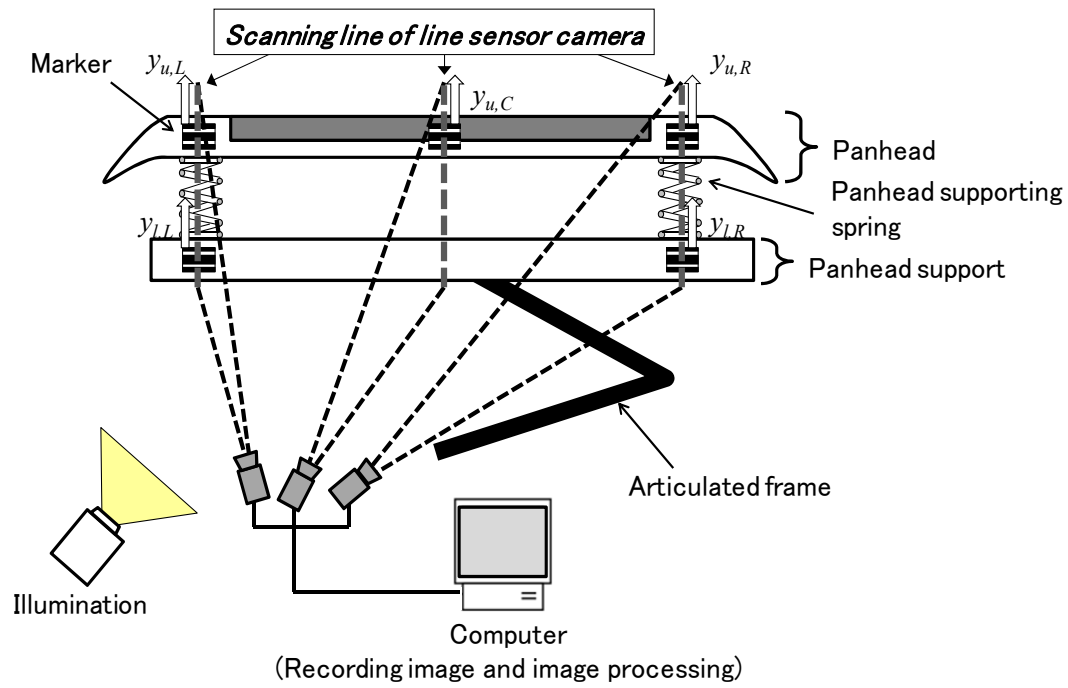

Figure 3: Measuring the contact force of pantograph by image processing technology. 
markers are taken by line sensor cameras with high time and spatial resolution mounted on the rooftop of the train near the pantograph. Illuminations installed near the pantograph help cameras to take clear images of the markers. Figure 4 shows a sample of image of the marker taken by the line sensor camera. This image consists of many time-ordered one-dimensional images captured by the line sensor camera.

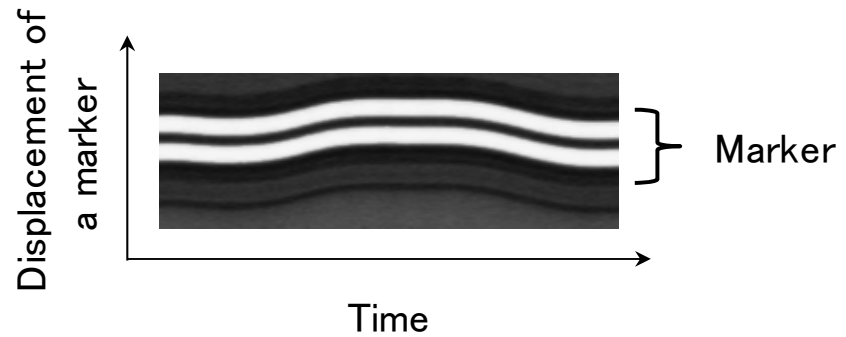

Figure 4: Images of a marker taken by a line sensor camera.

Symbols, $y_{u, L}, y_{l, L}, y_{u, R}, y_{l, R}$ and $y_{u, C}$ in the Figure 3 are vertical displacements of the markers. These displacements are obtained by the line sensor cameras using image processing technology with the pattern matching based on the normalized cross-correlation (NCC) method. NCC enhanced spatial resolution of displacement measurement. Figure 5 shows the displacement evaluated with/without NCC. Figure 5 indicates that the spatial resolution can be improved by using NCC.

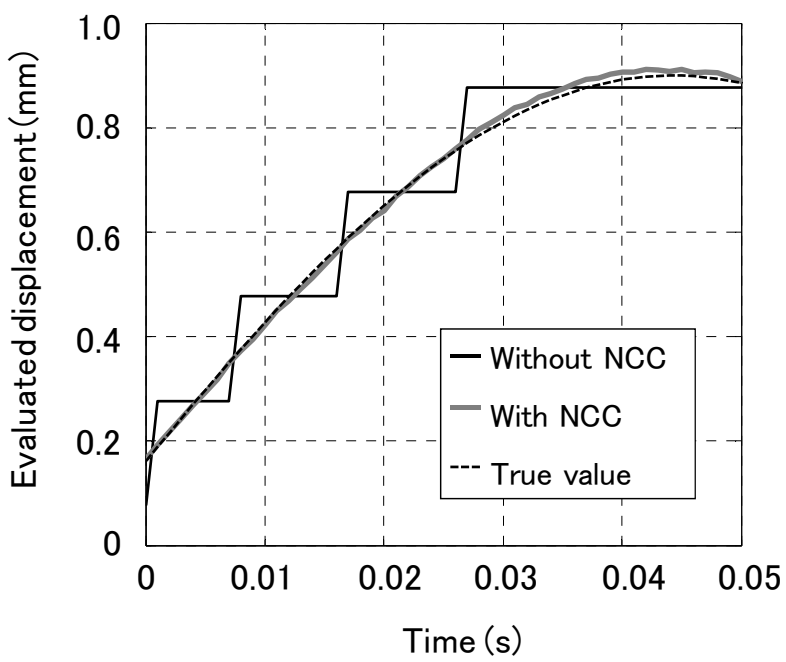

Figure 5: Displacement of a marker evaluated by NCC. 


\subsection{Measuring the contact force}

As mentioned before, the spring reaction forces $F_{b s}$ of the panhead supporting spring can be evaluated by the product of the spring coefficient and the spring deformation. The spring deformation can be taken by the image processing technologies as described in section 3.1. If the friction force between the panhead and the panhead support can be negligible, the inner force $F_{b}$ consists of only spring reaction forces $F_{b s}$.

Vertical accelerations $a_{u, L}, a_{u, R}$ and $a_{u, C}$ at the position of the markers can be obtained as the second derivatives of the displacements $y_{u, L}, y_{u, R}$ and $y_{u, C}$ with respect to time. The inertia force $F_{I}$ acting on the panhead is obtained by summing the products of the accelerations and the corresponding equivalent masses. The method for identification of the equivalent masses is described in the following.

The equivalent masses can be obtained by an excitation test. The inertia force $F_{I}$ can be estimated by eqn. (6):

$$
F_{I}=F_{c, \text { applied }}-F_{b}
$$

where, $F_{c, \text { applied }}$ and $F_{b}$ are excitation force and inner force, respectively. A frequency transfer function of $a_{u, i}(i=\mathrm{L}, \mathrm{C}, \mathrm{R})$ versus $F_{I}$ is expressed as follows:

$$
G_{i}(j \omega)=\hat{a}_{u, i} / \hat{F}_{I}
$$

where, $j$ and $\omega$ are imaginary unit and angular frequency. $\hat{a}_{u, i}$ indicates the Fourier transform of $a_{u, i}$. The frequency transform function can be written in matrix form:

$$
\mathbf{G}=\left(\begin{array}{ccc}
G_{L}\left(j \omega_{1}\right) & G_{C}\left(j \omega_{1}\right) & G_{R}\left(j \omega_{1}\right) \\
\vdots & \\
G_{L}\left(j \omega_{n}\right) & G_{C}\left(j \omega_{n}\right) & G_{R}\left(j \omega_{n}\right)
\end{array}\right)
$$

By using eqn. (8), the equivalent masses $m_{L}, m_{C}$ and $m_{R}$ for each marker are calculated as follows:

$$
\left(\begin{array}{lll}
m_{L} & m_{C} & m_{R}
\end{array}\right)^{T}=\left(\begin{array}{c}
\operatorname{Re}(\mathbf{G}) \\
\operatorname{Im}(\mathbf{G})
\end{array}\right)^{+} \mathbf{V}
$$

where, $\operatorname{Re}(\mathbf{G})$ and $\operatorname{Im}(\mathbf{G})$ indicate real part and imaginary part of $\mathbf{G}$, respectively. The superscript $T$ and + indicates transposed matrix and generalized inverse matrix, respectively. A vector $\mathbf{V}$ is expressed as follows:

$$
\begin{aligned}
\mathbf{V} & =\left(\begin{array}{llllll}
v_{1} & \cdots & v_{n} & v_{n+1} & \cdots & v_{2 n}
\end{array}\right)^{T} \\
v_{1} & =\cdots=v_{n}=1, v_{n+1}=\cdots=v_{2 n}=0
\end{aligned}
$$

As stated above, we can evaluate the inner force and the inertia force required to measure the contact force by using the image processing technology with line 
sensor cameras. Therefore, the contact force can be measured by substituting these forces and aerodynamic force in eqn. (1). Figure 6 indicates the measurement procedure of this method.

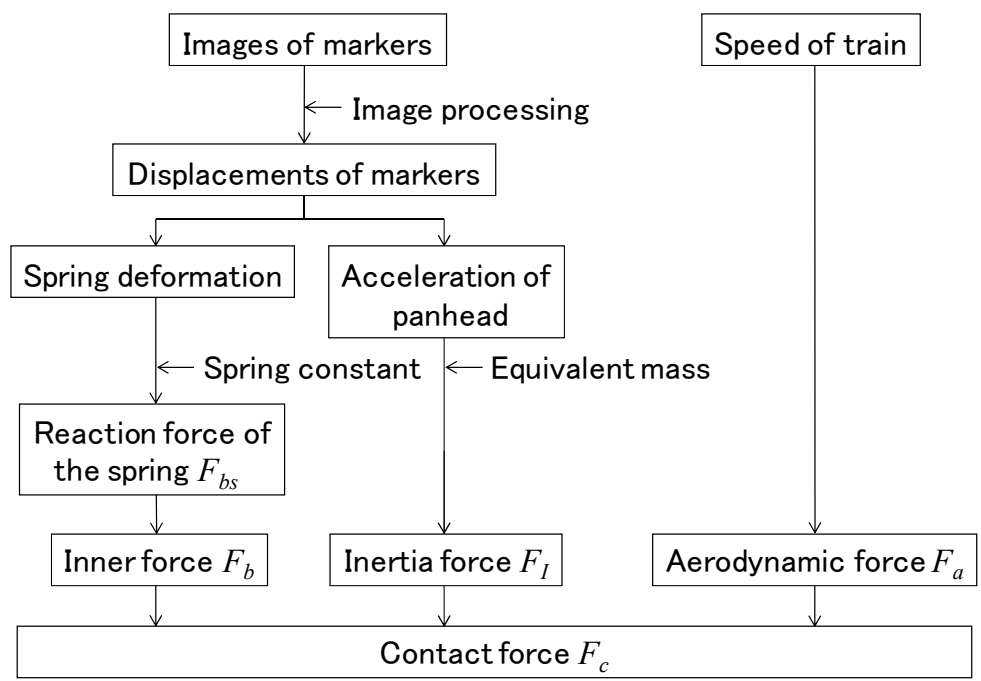

Figure 6: Procedure of the measurement method of the contact force by image processing technology.

\section{Verification test of the new method}

Authors carried out an excitation test of a pantograph in order to verify measurement accuracy of the new method. Figure 7 shows photo of the test.

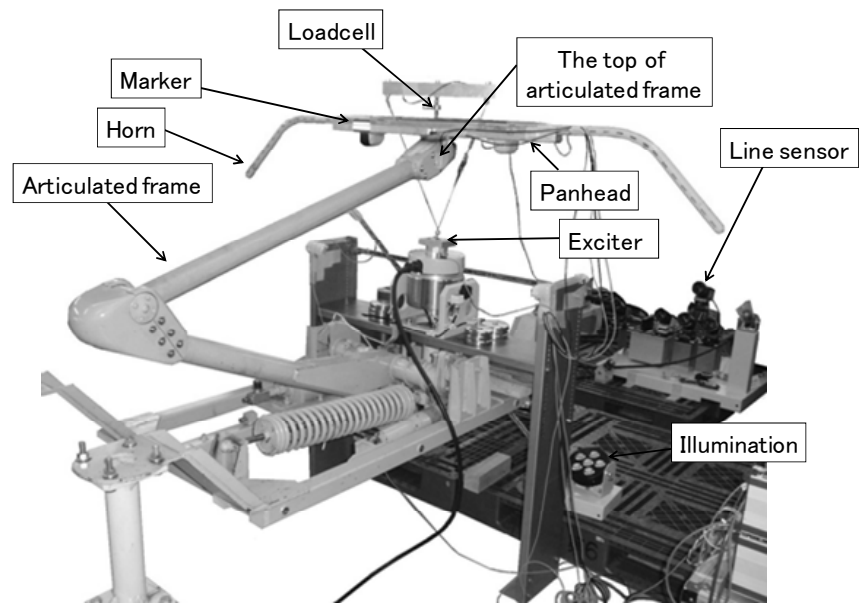

Figure 7: Photo of the excitation test of a pantograph. 
A single-arm type pantograph was used in the test. Friction force, which affected between the panhead and the panhead support of this pantograph, can be negligible.

An exciter was placed under the panhead and a loadcell was put on the panhead to measure excitation force $F_{c \text {,applied }}$. The excitation force $F_{c, \text { applied }}$ was compared with measured force $F_{c \text {,measured }}$ measured by the new method, which was identical to the excitation force ideally.

Two line sensor cameras (pixels: 7450pix, sampling frequency: $4.73 \mathrm{kHz}$ ) and illuminations were mounted near the pantograph. Three markers were set on the surfaces of the panhead and the panhead support (see Figure 8). One line sensor camera took images of two markers put on the surface of the panhead and the pantograph support to evaluate the spring reaction force of the panhead supporting springs and the inertia force of the panhead. The other line sensor camera took images of a marker put on the surface of the panhead to evaluate the inertia force of the panhead.

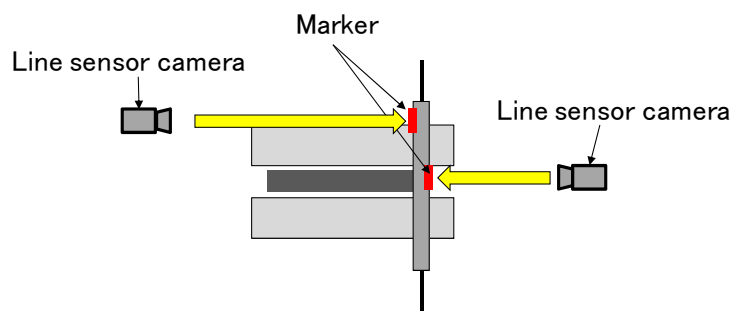

(a) overview

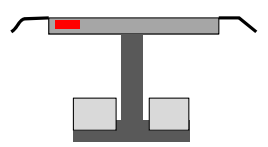

(b) side view

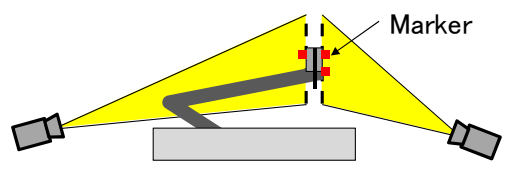

(c) front view

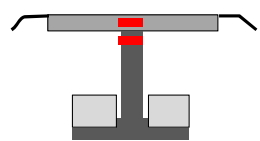

(d) side view

Figure 8: The position of line sensor cameras.

The test result is as shown in Figure 9. This figure indicates gain and phase of the frequency transfer function of the measured contact force $F_{c \text {,estimated }}$ versus the exciting force $F_{c, \text { applied }}$.

The European Standard (EN50317) provides the allowance of the measurement accuracy of a contact force measurement method calculated by using following formula [8]:

$$
\left\{1-\frac{1}{\left(f_{n}-f_{1}\right)} \sum_{k=1}^{n-1}\left(f_{k+1}-f_{k}\right)\left|1-\frac{\hat{F}_{c, \text { measured }}}{\hat{F}_{c, \text { applied }}}\right|\right\} \times 100 \%
$$


where, $f_{n}$ and $f_{l}$ is the maximum frequency and minimum frequency of the frequency range required to measure the contact force. We verified the measurement accuracy of the new method by using eqn. (11). Table 1 shows the accuracy of the new method and the accuracy required on the standard. It can be confirmed that the new contact force measurement method has satisfactory measurement accuracy, which meets a requirement of the standard.

Table 1: $\quad$ The measurement accuracy of the new method.

\begin{tabular}{|l|c|c|}
\hline \multirow{2}{*}{} & \multicolumn{2}{|c|}{ Measurement accuracy \% } \\
\cline { 2 - 3 } & $\begin{array}{l}\text { Measurement accuracy } \\
\text { of our method }\end{array}$ & $\begin{array}{l}\text { Required accuracy } \\
\text { on EN50317 }\end{array}$ \\
\hline Up to $10 \mathrm{~Hz}$ & 91.5 & $>80$ \\
\hline Up to $20 \mathrm{~Hz}$ & 90.1 & $>90$ \\
\hline
\end{tabular}

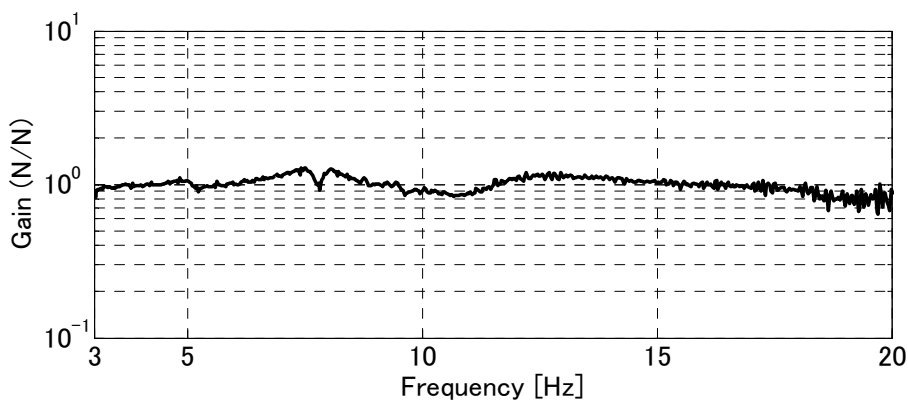

(a) Gain of transfer function $\hat{F}_{c \text {,measured }} / \hat{F}_{c \text {,applied }}$

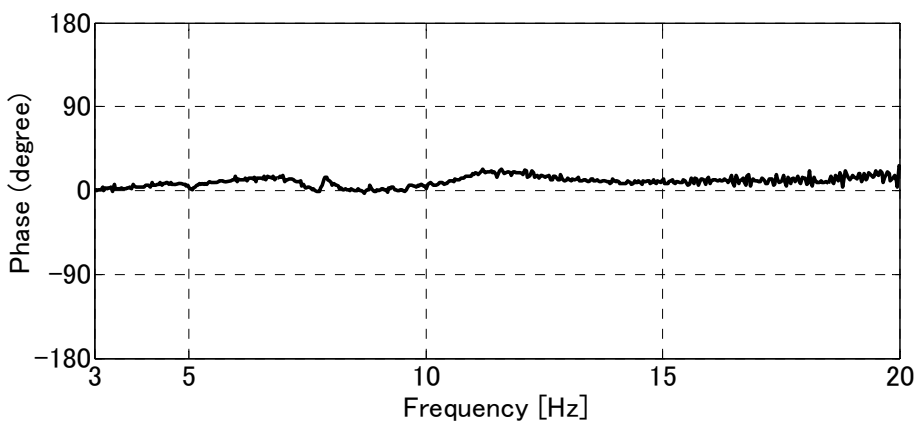

(b) Phase of transfer function $\hat{F}_{c, \text { measured }} / \hat{F}_{c \text {,applied }}$

Figure 9: The result of vilification test. 


\section{Conclusions}

The authors have developed the new contact force measurement method using the image processing technology, which needs no built-in sensor such as loadcells and so on. In order to verify measurement accuracy of this method, the excitation test was carried out. Consequently, it can be confirmed that the contact force measurement method has the satisfactory measurement accuracy, which meets requirement of EN50317.

\section{References}

[1] EN 50119:2001, Railway applications - Fixed installations - Electric traction overhead contact lines, CENELEC, 2001.

[2] EN 50367:2002, Railway applications - Current collection systems Technical criteria for the interaction between pantograph and overhead line (to achieve free access), CENELEC, 2002.

[3] Shunichi K., Takahiro F., Kazuyoshi N., Diagnosis of Overhead Contact Line based on Contact Force, Quarterly Report of RTRI, Vol.47, No.1, pp. 39-45, 2006.

[4] Mitsuru I., Sei N., Takayuki U., A precise contact force measuring method for overhead catenary system, WCRR 2001, 2001.

[5] Sung-Il S., Young-Hyun C., Jin-Yong M., Choon-Soo P., Kin-Hwan K., A Study on the Measurement of Contact Force of Pantograph on High Speed Train, ICCAS2005, Korea, 2005.

[6] Mitsuru I., A study on the method of measuring the contact force between pantograph and contact wire (1st report, Extension of the frequency range for contact force measurement by an improved method of evaluating), Transactions of the Japan Society of Mechanical Engineers, Ser. C, Vol. 70, No.694, 2004 (in Japanese).

[7] Mitsuru I., The Contact Force between Pantograph and Contact Wire -An Estimation Method Using the Inversion Technique-, Quarterly Report of RTRI, Vol.45, No.2, pp. 80-85, 2004.

[8] EN 50317:2002, Railway applications - Current collection systems Requirement for and validation of measurements of the dynamic interaction between pantograph and overhead contact line, CENELEC, 2002. 


\title{
An automated long range ultrasonic rail flaw detection system based on the support vector machine algorithm
}

\author{
S. Moustakidis ${ }^{1}$, V. Kappatos ${ }^{2}$, P. Karlsson ${ }^{1}$, C. Selcuk ${ }^{2}$, \\ K. Hrissagis ${ }^{1} \&$ T.-H. Gan ${ }^{2}$ \\ ${ }^{1}$ Centre of Research and Technology - Thessaly (CERETETH), \\ Biomechanics and Interdisciplinary Research, Trikala, Greece \\ ${ }^{2}$ Brunel Innovation Centre (BIC), Brunel University, UK
}

\begin{abstract}
This paper presents an automated long range ultrasonic (LRU) flaw detection system based on an effective pattern recognition approach. The method comprises three stages: 1) multiple feature extraction techniques were applied for better representation of the signals in time and frequency domain, 2) feature selection was employed next for feature ranking, according to discrimination power, and finally 3) the classification task was accomplished by means of a kernel-based support vector machine (SVM). For the training and validation of the algorithm, an extensive experimental investigation was carried out on the foot section of a $4.30 \mathrm{~m}$ long rail (CEN 56). Different depths of transversal slots have been induced in the foot at $3 \mathrm{~m}$ from the excitation point. The results show that the proposed method is able to effectively detect flaws.

Keywords: long range ultrasonic, support vector machine, flaw, detection.
\end{abstract}

\section{Introduction}

Trains constitute one of the most important and common transport means for people and goods. In 2010, throughout Europe more than 5,500 broken rail incidents were reported, exactly the same number as two years before in 2008 [1]. The catastrophic rail failure due to growth of structural flaws can be avoided by careful inspection and appropriately scheduled maintenance. There are several non destructive testing (NDT) techniques such as visual inspection, magnetic 
induction, eddy current, photo-thermal, and conventional ultrasonic testing, used for maintenance and inspection of the rail network [2,3]. However, current NDT methods are limited to detect flaws in the rail foot, especially in the side edges away from the web section [4]. Therefore, an alternative solution enable to inspect the completed foot section of rail would be greatly desirable.

LRU testing is a relatively recent technology being developed for rail inspection, which is used to overcome the previous difficulty of flaw detection in the rail foot section [5,6]. LRU testing relies on the use of ultrasonic guided wave modes in the kilohertz range (typically between 20 and $300 \mathrm{kHz}$ ) with relatively long ultrasonic wavelengths in comparison with conventional ultrasonic testing [7]. These waves propagate with low attenuation in media, covering large distance away from the ultrasonic source.

The received LRU signal usually consists of a number of peaks that correspond to reflections from features of the structure under inspection, such as crack, corrosion areas, welds etc. The several wave modes i.e. dispersive, the rail fastening features, the background noise etc. lead to significant reduction of the sensitivity and the resolution of flaw detection. Therefore, the detection of any flaw reflections requires us to apply sophisticated signal processing and pattern recognition techniques.

This paper presents an efficient system for automated flaw detection using a robust set of features which are extracted from the received LRU signal in time and frequency domain. The classification module was carried out using a kernelbased SVM algorithm. The rest of the paper is organized as follows: in section 2, a detailed description of the proposed method is given. Section 3 describes the experimental setup and procedure. The results of the evaluation process are discussed in section 4. A summary of conclusions is given in the last section.

\section{Proposed flaw recognition scheme}

To cope with the complex problem of flaw detection utilizing ultrasonic signals, the design of the recognition system should essentially involve the following three aspects: (1) feature extraction, (2) feature selection and (3) decision making. The problem domain dictates the choice of suitable pre-processing feature extraction/selection techniques and the classification model.

\subsection{Feature extraction}

It is well recognized that combining different types of characteristics (timedomain and frequency-domain) extracted from the ultrasonic signals can improve the detection capabilities of the applied technique. To this end, we consider four feature sets: first-order statistics from time domain, power spectral density (PSD) measures [8], short time Fourier transform (STFT) and wavelet features (WF) [9]. 


\subsubsection{First-order features (time domain)}

Features computed via first-order statistics will be used to capture the low-level information from time-domain. They are defined as given in the following:

$$
\begin{aligned}
\text { Maximum value: } & \operatorname{ma}=\frac{1}{N} \max _{n=0}^{N}\{x(n)\} \\
\text { Minimum value: } & \mathrm{mi}=\frac{1}{N} \min _{n=0}^{N}\{x(n)\} \\
\text { Average: } & \mathrm{m}=\frac{1}{N} \sum_{n=0}^{N} \mathrm{x}(n) \\
\text { Variance: } & \mathrm{v}=\frac{1}{N} \sum_{n=0}^{N}\{x(n)-m\}^{2} \\
\text { Energy: } & \mathrm{e}=\frac{1}{N} \sum_{n=0}^{N}|x(n)|^{2}
\end{aligned}
$$

\subsubsection{Power spectral density}

The power spectrum or the PSD of a random signal $\mathrm{x}(n)$ is defined as the Fourier transform of the autocorrelation function $r_{x x}(m)$. It is defined as follows:

where,

$$
P S D\{x(t)\}=S(\omega)=\sum_{m=-(N-1)}^{N-1} r_{x x}(m) \exp (-j \omega m)
$$

Another approach to simplifying the results of a power spectral analysis is to find parameters that describe particular characteristics of the spectrum distribution. The first of these descriptors that was used in our experiments is the peak power frequency (PPF), which is simply the frequency in a spectrum at which the highest power in that epoch occurs. The median power frequency (MPF) was also employed representing that frequency which bisects the spectrum, with half the power above and the other half below. Finally, the spectral edge frequency (SEF) was computed as a descriptive indicator extracted from the spectrum distribution. SEF is the highest frequency in the signal, that is, the high-frequency edge of the spectral distribution.

\subsubsection{Short time Fourier transform}

The STFT is defined as the discrete Fourier transform (DFT) applied to the "windowed" segments. The discrete STFT of a discrete-time signal $x(n)$ at time instant $n$ is defined as 


$$
\operatorname{STFT}\{x(t)\}=X(n, k)=\sum_{k=0,1,2, \ldots, N-1}^{N-1} x(n+m) W(m) \exp \left(-j \frac{2 \pi}{N} k m\right)
$$

where $n$ and $k$ are the discrete time and frequency variables, respectively. The preceding equation is interpreted as the Fourier transform of $x(n+m)$ as viewed through a window $W(m)$ that has a stationary origin and $n$ changes. The signal is shifted past the window so that at each $n$ a different portion of the signal is viewed. The low-level indicators defined in (1)-(5) will be computed on the STFT coefficients of each frequency level generating a time-frequency feature subset.

\subsubsection{Wavelet Transform (WT)}

The discrete wavelet transform uses a different basis than the STFT. The STFT uses complex sinusoids, with increasing frequency, which are shaped by the window function. Wavelet transforms, on the other hand, maintain the same number of oscillations within the window, but compress or dilate the window to adjust the frequency. Especially, the wavelet transform uses wide and narrow windows for slow and fast frequencies, respectively, thus leading to an optimal time-frequency resolution in all frequency ranges. The energy of the distorted signals partitioned at different resolution levels will be used for the flaw recognition task.

\subsection{Feature selection}

This task consists in identifying informative and non-redundant feature subsets. An efficient feature selection technique will be employed to reduce the highdimensionality of the recorded ultrasonic signal and provide a more descriptive and compact signal representation [10]. In addition to enhanced accuracy rates and dimensionality reduction, the method has reasonably low computational demands, being able to cope with the high-dimensional feature space.

The implemented technique performs forward selection guided by a novel fuzzy criterion, handling simultaneously both the discrimination power and the complementary characteristics between the features. The method relies on a local fuzzy evaluation measure with respect to patterns that reveal the adequacy of data coverage for each feature. Furthermore, feature selection is driven by a fuzzy complementary criterion which assures that those features are iteratively introduced, providing the maximum additional contribution with regard to the information content given by the previously selected features. To decide on the number of retained features, an efficient termination condition is utilized, thus avoiding the trial-and-error procedure usually employed in the literature.

\subsection{Classification}

Consider a data set comprising labelled training patterns: $D=\left\{\left(\boldsymbol{x}_{i}, y_{i}\right), i=1, \ldots, N\right\}$. Each pattern $\boldsymbol{x}_{i}=\left[x_{i, 1}, x_{i, 2}, \ldots, x_{i, n}\right]^{T} \in \mathfrak{R}^{n}$ belongs to one of two classes, with its class label given as $y_{i} \in\{+1,-1\}$. Given a nonlinear 
mapping $\Phi: \Re^{n} \rightarrow \mathcal{F}$, each vector $\boldsymbol{x}_{i}$ in the original feature space is transformed into a potentially higher dimensional feature space $\mathcal{F}: \boldsymbol{x}_{i} \rightarrow \Phi\left(\boldsymbol{x}_{i}\right)$, $i=1, \ldots, N$. The embedding of feature mapping and the associated kernel function lead to a powerful nonlinear scalar-product-based algorithm (K-SVM) [11], executed in $\mathcal{F}$. K-SVM seeks for a suitable separating hyperplane in the transformed space $\mathcal{F}: f(\boldsymbol{x})=((\boldsymbol{w} \cdot \Phi(\boldsymbol{x}))+b)=0$, parameterized by the pair $(\boldsymbol{w}, b), w \in \mathcal{F}, b \in \mathfrak{R}$. The optimal decision function is obtained by

$$
f(x)=\operatorname{sgn}\left(\sum_{i \in S} a_{i} y_{i} K\left(\boldsymbol{x}_{i}, \boldsymbol{x}\right)+b\right)
$$

where $S=\left\{i: 0<a_{i}^{*} \leq C\right\}$. Coefficient $a_{i}$ is non-zero when $\boldsymbol{x}_{i}$ is a support vector; otherwise it is zero. Any function satisfying the Mercer's theorem can be used as scalar product, thus serving as a kernel function. In this research, we employ the Gaussian RBF kernel:

$$
K\left(\boldsymbol{x}_{i}, \boldsymbol{x}_{j}\right)=\exp \left(\frac{-\left\|\boldsymbol{x}_{i}-\boldsymbol{x}_{j}\right\|^{2}}{2 \sigma^{2}}\right)
$$

where $\sigma$ denotes the variance along the feature axis. Selection of the RBF kernel is dictated by its ability to handle high-dimensional data. In our simulations, the parameters $C$ and $\sigma$ are heuristically determined from a grid of preselected values $C \in\{10,30,50,100\}$ and $\sigma \in\{0.005,0.01,0.1,0.5,1\}$.

Since K-SVMs were originally designed for binary classification, a decomposition scheme should be devised to tackle multiclass problems. Oneversus-all (OVA) is a common approach, accomplished by combining several binary SVM classifiers. For an M-class problem, OVA proceeds to constructing a set of binary classifiers $\left\{f_{1}, \ldots, f_{k}, \ldots, f_{M}\right\}$. Each $f_{k}, k=1, \ldots, M$, is trained individually to separate class $c_{k}$ from the rest of the classes, included in $\bar{C}_{k}=\{1, . ., \ell, . ., M \mid \ell \neq k\}$. Following the winner-takes-all principle, an unknown pattern $\boldsymbol{x}$ is then assigned to the class that exhibits the maximum decision function value $f_{k}(\boldsymbol{x})$ :

$$
c_{k}=\underset{k}{\arg \max }\left\{f_{k}(\boldsymbol{x})=\sum_{i \in S} a_{i}^{*} y_{i} K\left(\boldsymbol{x}_{i}, \boldsymbol{x}\right)+b^{*}\right\}
$$

\section{Experimental procedure}

The commercial guided wave ultrasonic system Teletest ${ }^{\circledR}[12]$ was used to excite and to collect the signal responses from a $4.30 \mathrm{~m}$ length specimen of rail (CEN 56). The piezo-electric elements were mounted (bonded) at one end of the rail specimen. According to previous modelling work [5], the arrays of 
transducers were displaced by $37.3 \mathrm{~mm}$ from each other in order to enhance the wave propagation in one direction.

The first set of experiments has been carried out in a rail with non-flaw. A sine signal that has been modulated by a Hanning window, reducing the transmitted bandwidth, with the excitation frequency sweep from 50 to $120 \mathrm{KHz}$ (with a step of $5 \mathrm{KHz}$ ), and for different number of cycle pulse was used to excite the ultrasonic transducers. The pulse has travelled through the rail and reflected back from the rail end. The echo was recorded at a distance of approximately $8.6 \mathrm{~m}$ (i.e. $2 \mathrm{x}$ rail length).

Subsequently, a cut was introduced in the foot with different depths. Especially, transverse slots were cut on the one side of the rail foot (figure 1) at different depths. These slots have been induced in the foot at $3 \mathrm{~m}$ from the excitation point with depth varying from $1 \mathrm{~mm}$ to $4 \mathrm{~mm}$. Table 1 summarises the dimensions of the different flaw sizes.

Table 1: $\quad$ Slot sizes in the foot section of the rail.

\begin{tabular}{|c|c|c|c|c|}
\hline $\begin{array}{c}\text { Slot depth } \\
(\mathrm{mm})\end{array}$ & $\begin{array}{c}\text { Slot length } \\
(\mathrm{mm})\end{array}$ & $\begin{array}{c}\text { Slot width } \\
(\mathrm{mm})\end{array}$ & $\begin{array}{c}\text { Slot area } \\
\left(\mathrm{mm}^{2}\right)\end{array}$ & $\begin{array}{c}\text { Loss of cross- } \\
\text { sectional area (\%) }\end{array}$ \\
\hline 1 & 17 & 1 & 17 & 0.60 \\
\hline 2 & 18 & 1 & 36 & 1.27 \\
\hline 4 & 16 & 1 & 64 & 2.27 \\
\hline
\end{tabular}

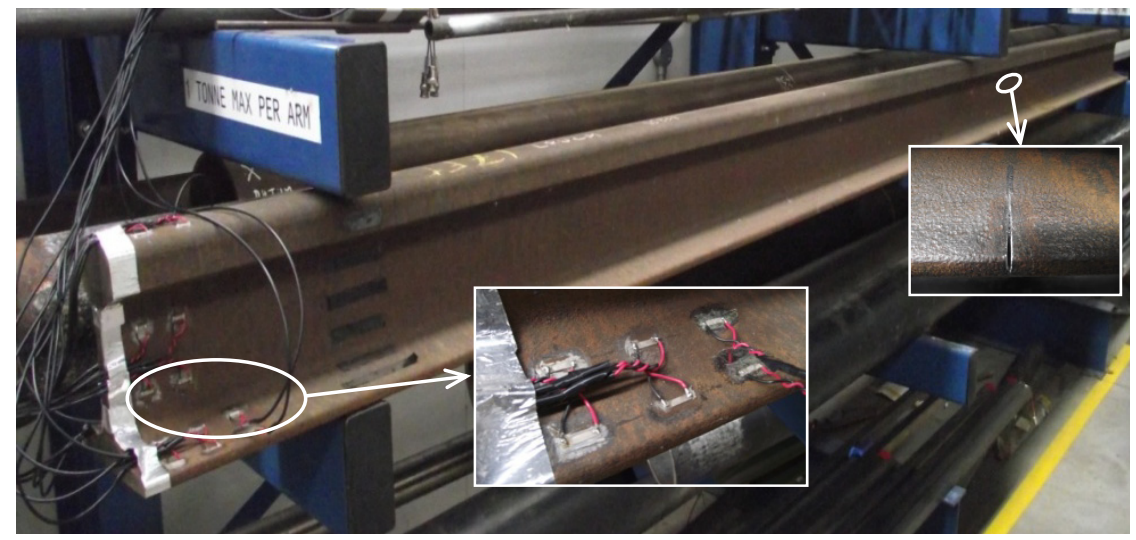

Figure 1: The $4.30 \mathrm{~m}$ length rail specimen, the mounted piezo-electric elements at the one end of rail and the position of flaws.

All the experiments have been repeated (64 times) and averaged to minimise the effects of any random noise. The following figures present the pulse-echo response (average of 64 times) using $100 \mathrm{KHz}$ centre excitation frequency, of the one transducer mounted on the furthest size edge from the web section, at presence of $1 \mathrm{~mm}$ (a), $2 \mathrm{~mm}$ (b), and $4 \mathrm{~mm}$ (c) depth slot, respectively. 
The dead zone, caused by the hardware's inability to transmit signals at the same time as receiving signals, is hidden in these figures. There is also some ringing seen immediately after this zone, which probably is an artefact of the test caused by transducers resonating after they have been excited and ringing down'.

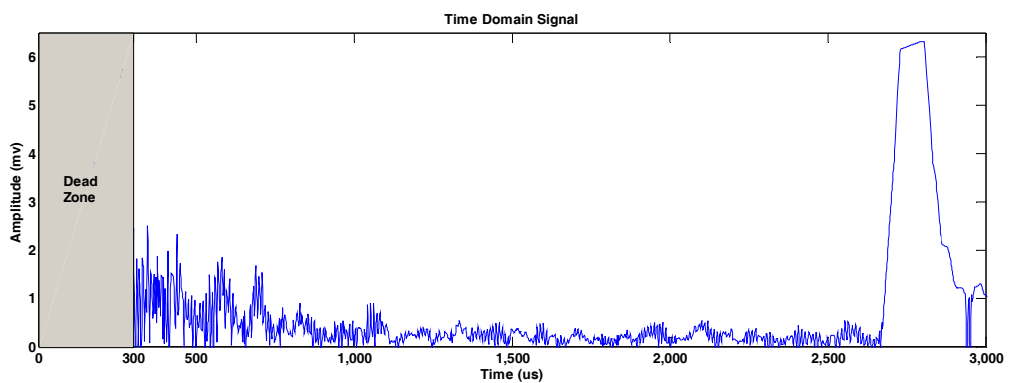

(a)

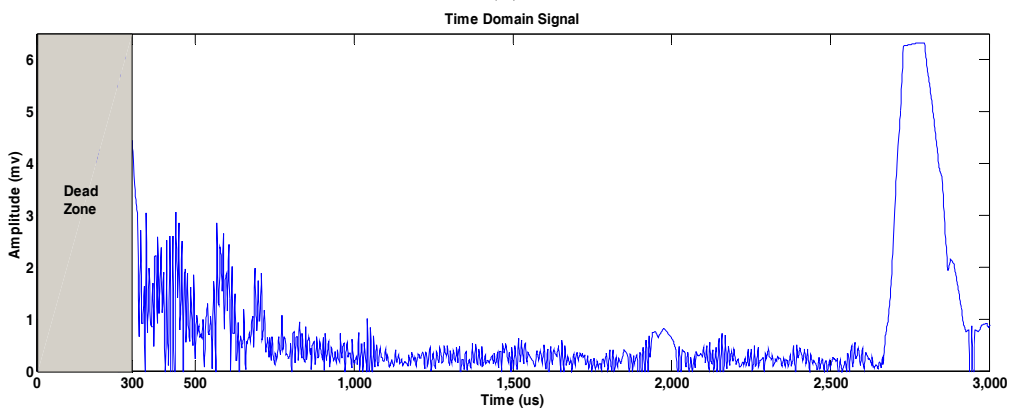

(b)

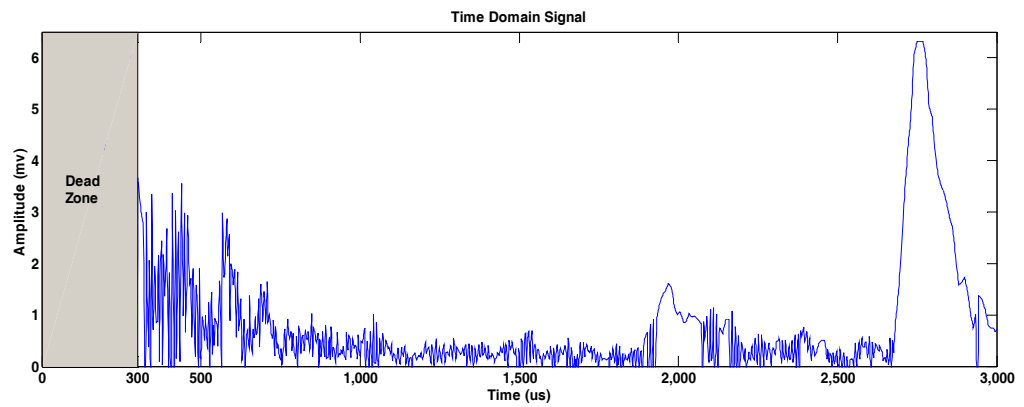

(c)

Figure 2: $\quad$ The pulse-echo response for $1 \mathrm{~mm}(\mathrm{a}), 2 \mathrm{~mm}$ (b), and $4 \mathrm{~mm}$ (c) depth slot, respectively. 


\section{Detection results}

In this section, we demonstrate the efficiency of the suggested flaw recognition scheme on the acquired LRU signals. Following 10-fold cross-validation, the original sample is randomly partitioned into 10 subsamples. A single subsample is firstly retained for testing the classification model, and the remaining 9 subsamples are used as training data. The cross-validation process is totally repeated 10 times (number of the folds), with each of the 10 subsamples used exactly once as the testing data. The training and testing performances are then determined by averaging the respective classification rates over the ten different folds.

The proposed classification process is described as follows:

1. For each training set, the proposed feature extraction techniques are applied generating: (i) first-order statistics from time domain, (ii) PSD measures, (iii) STFT and (iv) WF.

2. Features are initially normalized by their standard deviations to treat attribute ranges on a common scale.

3. The proposed feature selection technique is implemented to select the optimal feature subspace comprising the most informative and complementary features.

4. The selected feature subset is classified by K-SVM. The training and testing performances are determined by averaging the respective classification rates over the ten folds.

To obtain a better visualization of the classification performance, confusion matrix will be also used as an error measure, where each column of the matrix represents the instances in an actual class, while each row represents the instances in an a predicted class. The final performance will be evaluated based on the overall accuracy (OA) and the class accuracies (CA) derived from the confusion matrix.

\subsection{Flaw detection}

The problem of flaw detection was initially investigated, defining a binary classification problem that comprises the acquired LRU signals organized into two data classes as given in Table 2. The aforementioned classification process was implemented to differentiate the baseline signals (C1) from the signals collected from the rail after introducing the flaws (C2).

Table 2: $\quad$ Dataset description for the flaw detection problem.

\begin{tabular}{|c|c|c|c|}
\hline Class & Description & Content & $\begin{array}{c}\text { Number of } \\
\text { Patterns }\end{array}$ \\
\hline C1 & non-flaw & LRU signals collected from a non-flaw rail & 64 \\
\hline C2 & flaw & $\begin{array}{c}\text { LRU signals collected from a rail with } \\
\text { slots (1mm, 2mm and 4mm) }\end{array}$ & 48 \\
\hline
\end{tabular}

Figure 3 shows the average classification rates versus the number of selected features for the training (dashed line) and testing datasets (solid line). The use of 
the proposed analysis provides an almost exponentially increase of classification rates in both the training and testing data sets. The recognition performance starts from a high level of $90.15 \%$ and the classification ability further rises as the number of features increases. When the feature number is 4 , the average rates reach a threshold of $98.21 \%$, which suggests that considering the first 4 features incorporates the major part of information contained in the LRU signals. The inclusion of additional features does not increase the classification scores. Table 1 represents the error confusion matrix in the flaw detection problem for the best feature subset obtained (4 first selected features). The minimal confusion between the two classes and the almost perfect CA rates observed imply that the proposed classification method is capable to detect successfully the flaw present in the rail, accomplishing fine recognition accuracies.

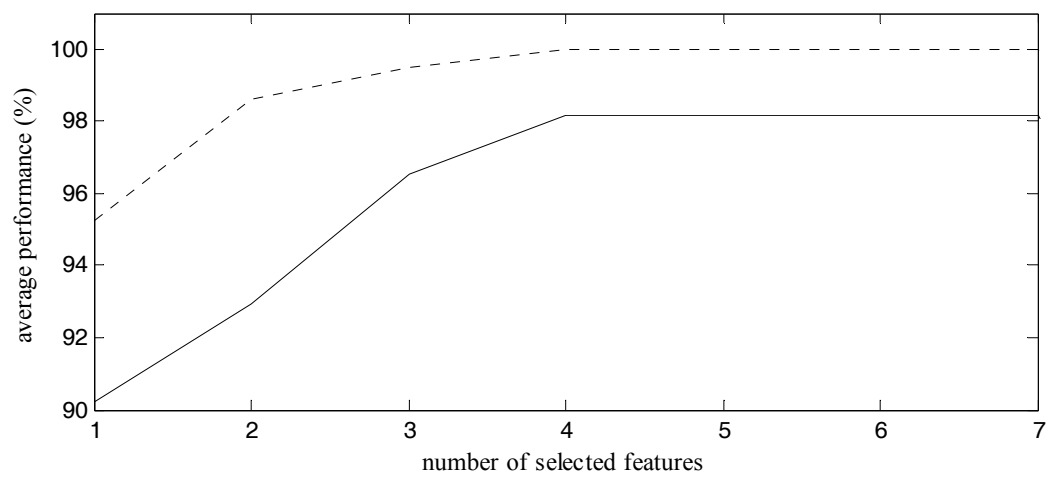

Figure 3: Average classification rates versus the number of features for the training (dashed line) and checking sets (solid line).

\subsection{Flaw severity estimation}

To qualify the efficiency of our approach in recognizing the flaw severity, we applied the proposed scheme in the multi-class dataset comprising the following three classes: C2a: LRU signals that correspond to $1 \mathrm{~mm}$ flaw, C2b: LRU signals that correspond to $2 \mathrm{~mm}$ flaw and C2c: LRU signals that correspond to $4 \mathrm{~mm}$ flaw. After verifying in Section 4.1 the classification capabilities of the suggested approach in differentiating the baseline signals with flaws, we proceed exploring its capabilities to discriminate the three different flaw sizes (1, 2 and $4 \mathrm{~mm})$.

Table 3: $\quad$ Error confusion matrix in flaw detection for the best feature subset obtained.

\begin{tabular}{|c|c|c|c|}
\hline & C1: non-flaw & C2: flaw & \\
\cline { 1 - 3 } C1: non-flaw & 62 & 0 & \\
\hline C2: flaw & 2 & 48 & total accuracy (\%) \\
\hline CA $(\%)$ & 96.88 & 100 & 98.21 \\
\hline
\end{tabular}


Table 4 represents the error confusion matrix in the flaw severity recognition problem where no satisfactory rates are obtained for the overall accuracy (OA) and per-class accuracies (CA). Small $\mathrm{CA}$ rates are given for $\mathrm{C} 2 \mathrm{a}$ and $\mathrm{C} 2 \mathrm{~b}$ (43.75\% and $68.75 \%$ respectively), revealing significant confusion between the predicted labels of the classifier for the signals with flaw sizes of 1 and $2 \mathrm{~mm}$. The largest flaw size $(4 \mathrm{~mm})$ seems to be identified with a higher CA rate of $87.50 \%$ and smaller confusion with the other two classes. The overall accuracy of $66.67 \%$ verifies the general inability of the model to identify the flaw severity, especially in the case of the smaller flaw diameter.

Table 4: $\quad$ Error confusion matrix in flaw severity discrimination.

\begin{tabular}{|c|c|c|c|c|}
\hline & C2a: $1 \mathrm{~mm}$ & C2b: $2 \mathrm{~mm}$ & C2c: $4 \mathrm{~mm}$ & \multirow{2}{*}{} \\
\cline { 1 - 4 } C2a: $1 \mathrm{~mm}$ & 7 & 5 & 1 & \\
\hline C2b: $2 \mathrm{~mm}$ & 5 & 11 & 1 & \\
\hline C2c: $4 \mathrm{~mm}$ & 4 & 0 & 14 & total accuracy $(\%)$ \\
\hline CA $(\%)$ & 43.75 & 68.75 & 87.50 & 66.67 \\
\hline
\end{tabular}

\subsection{Hierarchical flaw recognition}

A hierarchical classification technique is finally developed to cope with the problem of flaw detection and accurate flaw severity recognition. This approach exploits both the efficient computation of the hierarchical tree structure and the classification accuracy of SVMs. In the proposed two-level hierarchical classification method the whole SVM-based classification process, as described at the beginning of Section 4, is applied in two different levels:

1. Level 1 (flaw detection level): class $\mathrm{C} 1$ (non-flaw) is separated from class C2 (flaw),

2. Level 2 (flaw severity recognition level): class $\mathrm{C} 2 \mathrm{c}(4 \mathrm{~mm}$ flaw) is discriminated from the group of classes C2a (1mm flaw) and C2b (2mm flaw).

Due to the observed significant confusion between them, C2a and $\mathrm{C} 2 \mathrm{~b}$ are merged together forming a new meta-class that represents the category of signals collected from a rail with a small flaw size. Figure 4 depicts the proposed hierarchical classification algorithm, where each internal node corresponds to the

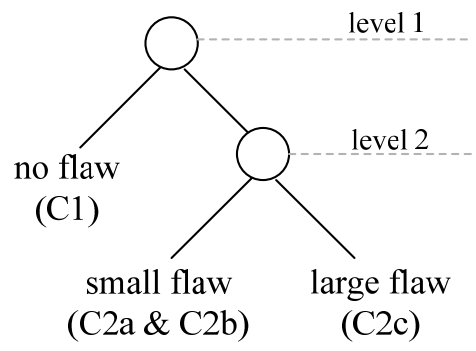

Figure 4: Proposed hierarchical classification model for flaw detection and severity assessment. 
applied classification model and the leaf-nodes correspond to the data classes considered.

Table 5 reveals the confusion between the three classes and cites the overall classification (OA) and class accuracies of the proposed approach applied on the hybrid problem of flaw detection and severity assessment in rails via the use of LRU signals. The technique accomplishes adequately high OA rate $(91.96 \%)$, whereas its flaw detection capabilities at level 1 remain almost perfect $(96.88 \%)$. At the level of flaw severity assessment (level 2), the class (C2a and C2b) that corresponds to small flaws is recognized with a high confidence (93.75\%) whereas the large-flaw class $(\mathrm{C} 2 \mathrm{c})$ receives the moderate accuracy rate of $68.75 \%$.

Table 5: $\quad$ Error confusion matrix in flaw recognition.

\begin{tabular}{|c|c|c|c|c|}
\hline & $\begin{array}{c}\text { Non- } \\
\text { flaw }\end{array}$ & $\begin{array}{c}\text { Small } \\
\text { flaw }\end{array}$ & $\begin{array}{c}\text { Large } \\
\text { flaw }\end{array}$ & \multirow{2}{*}{} \\
\cline { 1 - 4 } non-flaw & 62 & 0 & 0 & \\
\cline { 1 - 4 } small flaw (1 and 2 mm) & 1 & 30 & 5 & \\
\hline large flaw (4mm) & 1 & 2 & 11 & total accuracy (\%) \\
\hline CA (\%) & 96.88 & 93.75 & $68.75 \%$ & 91.96 \\
\hline
\end{tabular}

\section{Discussions}

An automated LRU flaw detection system is suggested in this paper based on an effective pattern recognition approach. The most significant step consists of extracting and selecting distinguishing features, which is a task achieved by combining different feature extraction techniques and a novel computationally efficient feature selection. Classification was accomplished using a K-SVM, which performs state-of-the-art accuracy. The effectiveness of the proposed scheme is verified through comprehensive experimentation. The accuracy of the classification results suggests that this sophisticated method can be successfully used for rail flaw detection and severity assessment increasing the sensitivity detection and decreasing the depth flaw detection to $1 \mathrm{~mm}$.

In addition, our proposed system can be efficiently used, not only for rail inspection but also for other large and complex infrastructures such as ship hull, bridges etc. From the industrial point of view, the development of fast and efficient monitoring system can be easily achieved using few features in existing hardware.

In the future a wider range of sizes, locations, and types of flaws have to be studied. Further, the influence of fastening features such as toe insulator, side post insulator, rail pad, shoulder, positive lock-in and clip on rail will be also studied. Characterisation of the rail vibration induced by train passage will be also be helpful to understand the effect of guided wave propagation under such vibration conditions. 


\section{Acknowledgements}

This work was undertaken as part of the European MONITORAIL project which is a collaboration between the following organisations: TWI Ltd, Vermon SA, OpenPattern, Aerosoft S.p.A, Jackweld Ltd, Network Rail Infrastructure Ltd, Cereteth and Brunel University. The Project is co-ordinated by TWI Ltd. and is partly funded by the EC under the Collaborative project programme - Research for SMEs \& Research for SME Associations. Grant Agreement Number. 262194.

\section{References}

[1] Railway Safety Performance in the European Union, European Railway Agency, 2012.

[2] Papaelias, M. Ph., Roberts, C. and Davis, C. L., A review on nondestructive evaluation of rails: State-of-the-art and future development, Proceedings of the Institution of Mechanical Engineers, Part F: Journal of Rail and Rapid Transit, 222 (4), pp. 367-384, 2008.

[3] Kudinov, D. and Shaydurov, G., Non-contact nondestructive rail testing, International Siberian Conference on Control and Communications (SIBCON), pp. 290-295, 2009.

[4] Monitorail Deliverable-D1.1., Project requirements and specifications preparation of materials, 2011.

[5] Gharaibeh, Y., The application of guided waves for non-destructive examination of complex structures, Doctoral Dissertation, School of Engineering and Design, Brunel University, UK, 2011.

[6] Campos-Castellanos, C. Gharaibeh, Y. Mudge, P. and Kappatos, V., The application of long range ultrasonic testing (lrut) for examination of hard to access areas on railway tracks, 5th IET conference on Railway Condition Monitoring and Non-Destructive Testing, Derby, UK, 2011.

[7] Mudge, P., Practical enhancement achievable in long range ultrasonic testing by exploiting the properties of guided waves, 16th World Conference of Non-Destructive Testing, Montreal, Canada, 2004.

[8] Proakis, J.G. and Manolakis, D.G., Digital Signal Processing: Principles, Algorithms, and Applications, (2nd edition), New York: MacMillan, 1992.

[9] Mallat, G., Theory for multiresolution signal decomposition: the wavelet representation. IEEE Transactions on Pattern Analysis and Machine Intelligence, 11, pp. 674-693, 1989.

[10] Moustakidis, S.P. and Theocharis, J.B., SVM-FuzCoC: A novel SVMbased feature selection method using a fuzzy complementary criterion. Pattern Recognition, 43 (11), pp. 3712-3729, 2010.

[11] Vapnik VN., Statistical learning theory, New York: Wiley, 1998.

[12] Plant Integrity, http://www.plantintegrity.com/ 